\title{
The holomorphy conjecture for nondegenerate surface singularities
}

\author{
Wouter Castryck, Denis Ibadula and Ann Lemahieu*
}

\begin{abstract}
The holomorphy conjecture states roughly that Igusa's zeta function associated to a hypersurface and a character is holomorphic on $\mathbb{C}$ whenever the order of the character does not divide the order of any eigenvalue of the local monodromy of the hypersurface. In this article we prove the holomorphy conjecture for surface singularities which are nondegenerate over $\mathbb{C}$ w.r.t. their Newton polyhedron. In order to provide relevant eigenvalues of monodromy, we first show a relation between the normalized volume (which appears in the formula of Varchenko for the zeta function of monodromy) of faces in a simplex in arbitrary dimension. We then study some specific character sums that show up when dealing with false poles. In contrast with the context of the trivial character, we here need to show fakeness of certain poles in addition to the candidate poles contributed by $B_{1}$-facets.
\end{abstract}

\section{Introduction}

Let $K$ be a finite extension of the field of $p$-adic numbers $\mathbb{Q}_{p}$. Let $R$ be the valuation ring of $K$ and $P$ its maximal ideal. Suppose that the residue field $R / P$ has cardinality $q$. For $z \in K$, let $\operatorname{ord}(z) \in \mathbb{Z} \cup\{\infty\}$ denote its valuation, $|z|=q^{-\operatorname{ord}(z)}$ its absolute value and $\operatorname{ac}(z)=z \pi^{-\operatorname{ord}(z)}$ its angular component, where $\pi$ is a fixed uniformizing parameter for $R$.

Let $f(\underline{x}), \underline{x}:=\left(x_{1}, \ldots, x_{n}\right)$, be a non-constant polynomial over $K$, and $\chi: R^{\times} \rightarrow \mathbb{C}^{\times}$ a multiplicative character of $R^{\times}$, i.e. a homomorphism with finite image. We formally put $\chi(0)=0$. Let $Z_{f, 0}(\chi, K, s)$, resp. $Z_{f}(\chi, K, s)$, be the corresponding local Igusa zeta function, resp. global Igusa zeta function, i.e. the meromorphic continuation to $\mathbb{C}$ of the integral function

$$
Z_{0}(s)=\int_{P^{n}} \chi(\operatorname{ac}(f(\underline{x})))|f(\underline{x})|^{s}|d(\underline{x})|, \quad \operatorname{resp} . Z(s)=\int_{R^{n}} \chi(\operatorname{ac}(f(\underline{x})))|f(\underline{x})|^{s}|d(\underline{x})|,
$$

for $s \in \mathbb{C}$ with $\operatorname{Re}(s)>0$, where $|d(\underline{x})|=\left|d x_{1} \wedge \ldots \wedge d x_{n}\right|$ denotes the Haar measure on $K^{n}$ normalized such that the measure of $R^{n}$ is 1 .

For $f$ a polynomial over $R$, the local and global Igusa zeta function can be described in terms of solutions of congruences. For $i \in \mathbb{N}_{>0}$ and $u \in R / P^{i}$, let $M_{0, i}(u)$ and $M_{i}(u)$ be the numbers of solutions of $f(\underline{x}) \equiv u \bmod P^{i}$ in $\left(P / P^{i}\right)^{n}$ resp. $\left(R / P^{i}\right)^{n}$. Let $c$ be

\footnotetext{
${ }^{*}$ The research was partially supported by MCI-Spain grant MTM2010-21740-C02, by the ANR 'SUSI' project (ANR-12-JS01-0002-01) and by the research project G093913N of the Research Foundation - Flanders (FWO).
} 
the conductor of $\chi$, i.e. the smallest $a \in \mathbb{N}_{>0}$ such that $\chi$ is trivial on $1+P^{a}$. Then

$$
\begin{gathered}
Z_{0}(s)=\sum_{i=0}^{\infty} \sum_{u \in\left(R / P^{c}\right)^{\times}} \chi(u) M_{0, i+c}\left(\pi^{i} u\right) q^{-n(i+c)} q^{-i s}, \quad \text { and } \\
Z(s)=\sum_{i=0}^{\infty} \sum_{u \in\left(R / P^{c}\right)^{\times}} \chi(u) M_{i+c}\left(\pi^{i} u\right) q^{-n(i+c)} q^{-i s} .
\end{gathered}
$$

Igusa showed that these functions are rational functions in $q^{-s}$ and he gave a formula for $Z_{f, 0}(\chi, K, s)$ and $Z_{f}(\chi, K, s)$ in terms of an embedded resolution $(Y, h)$ of $f^{-1}\{0\}$ over $K$ (see [I] ). Let $E_{j}, j \in T$, be the (reduced) irreducible components of $h^{-1}\left(f^{-1}\{0\}\right)$, and let $N_{j}$, resp. $\nu_{j}-1$, be the multiplicity of $E_{j}$ in the divisor of $f \circ h$, resp. $h^{*}\left(d x_{1} \wedge \ldots \wedge d x_{n}\right)$ on $Y$. Then the poles of $Z_{f, 0}(\chi, K, s)$ and $Z_{f}(\chi, K, s)$ are among the values

$$
s=\frac{-\nu_{j}}{N_{j}}+\frac{2 k \pi i}{N_{j} \log (q)}, \quad k \in \mathbb{Z}, j \in T
$$

for which the order of $\chi$ divides $N_{j}$.

Let now $f \in F[\underline{x}]$, with $F \subset \mathbb{C}$ a number field, and let $K$ be a non-archimedean completion of $F$, i.e. a completion w.r.t. a finite prime. Let $R$ be its valuation ring and let $\chi: R^{\times} \rightarrow \mathbb{C}^{\times}$be a character. Then the poles of $Z_{f, 0}(\chi, K, s)$ and $Z_{f}(\chi, K, s)$ seem to be related to various invariants in singularity theory, such as the eigenvalues of monodromy and the roots of the Bernstein-Sato polynomial (see for example [D2]) and such as the jumping numbers (see for example [ST]). In this article we explore another connection conjectured by Denef, called the holomorphy conjecture. It follows from (11) that when the order of $\chi$ divides no $N_{j}$ at all, then the zeta functions $Z_{f, 0}(\chi, K, s)$ and $Z_{f}(\chi, K, s)$ are holomorphic on $\mathbb{C}$. Now, the $N_{j}$ are not intrinsically associated to $f^{-1}\{0\}$; however the order (as root of unity) of any eigenvalue of the local monodromy on $f^{-1}\{0\}$ divides some $N_{j}$, and those eigenvalues are intrinsic invariants of $f^{-1}\{0\}$. This observation inspired Denef to propose the following ([D2, Conjecture 4.4.2]):

Conjecture 1 (Holomorphy conjecture). For almost all non-archimedean completions $K$ of $F$ (i.e. for all except a finite number) and all characters $\chi$, the local (resp. global) Igusa zeta function $Z_{f, 0}(\chi, K, s)$ (resp. $\left.Z_{f}(\chi, K, s)\right)$ is holomorphic, unless the order of $\chi$ divides the order of some eigenvalue of the local monodromy of $f$ at some complex point of $f^{-1}\{0\}$.

This conjecture has been proven by Veys in [Ve, Theorem 3.1] for plane curves, and in [DV] Denef and Veys got a Thom-Sebastiani type result. In [RV] Rodrigues and Veys make several progresses on the holomorphy conjecture for homogeneous polynomials. Veys and the third author confirmed the conjecture for surfaces that are general for a toric idealistic cluster (see [LV, Theorem 24]). In [LVP1] the holomorphy conjecture has been introduced for ideals and was proven for ideals in dimension two.

In this article we prove the holomorphy conjecture for surface singularities that are nondegenerate over $\mathbb{C}$ w.r.t. their Newton polyhedron at the origin. In Section 2 we recall this notion, along with explicit formulas for the zeta functions in this context. By a formula of Varchenko the normalized volume of a face gets a key role in the 
search for eigenvalues of monodromy for nondegenerate singularities. In Section 3 we prove some properties on the normalized volume of faces in a simplex of arbitrary dimension. These properties might be of independent interest. We can use them in Subsection 5.1 to obtain a set of eigenvalues that is relevant for the holomorphy conjecture. Furthermore, we prove that some candidate poles of $Z_{f, 0}(\chi, K, s)$ (resp. $\left.Z_{f}(\chi, K, s)\right)$ are no actual poles. In [BV] some configurations of $B_{1}$-facets that give rise to false poles have been treated in the context of non-trivial characters. Actually, it seems that almost all configurations of $B_{1}$-facets give rise to fake poles (see Subsection 5.2 for the exact statement). We even find a configuration without $B_{1}$-facets where we need to show that the candidate pole is a false pole. These computations rely on the study of some specific character sums (see Section 4). We can then complete our proof using a nondegeneracy argument (see Lemma 2) which was used for the first time in [LVP2].

As a preliminary remark, we note that for the purpose of proving the holomorphy conjecture one can assume that

- $f$ has coefficients in the ring of integers $\mathcal{O}_{F}$ of $F$; indeed, multiplying $f$ by a constant $a \in F$ affects $Z_{f, 0}(\chi, K, s)$ and $Z_{f}(\chi, K, s)$ only for the completions $K$ in which $\operatorname{ord}(a) \neq 0$, of which there are finitely many,

- $\chi$ is a non-trivial character with conductor equal to 1 ; indeed, Denef proved that for almost all non-archimedean completions $K$ of $F$, if $\chi: R^{\times} \rightarrow \mathbb{C}^{\times}$is a multiplicative character which is non-trivial on $1+P$, then $Z_{f, 0}(\chi, K, s)$ and $Z_{f}(\chi, K, s)$ are constant on $\mathbb{C}$ (see [D2, Theorem 3.3]).

From now on we will just write $Z_{f, 0}(\chi, s)$ (resp. $\left.Z_{f}(\chi, s)\right)$ for $Z_{f, 0}(\chi, K, s)$ (resp. $\left.Z_{f}(\chi, K, s)\right)$.

\section{Nondegenerate singularities and their zeta functions}

\subsection{Nondegenerate singularities}

Assume that $f(\underline{x}) \in \mathcal{O}_{F}[\underline{x}]$ is a non-constant polynomial satisfying $f(\underline{0})=0$. Write

$$
f(\underline{x})=\sum_{\underline{k} \in \mathbb{Z}_{\geq 0}^{n}} c_{\underline{k}} \underline{x}^{\underline{k}},
$$

where $\underline{k}=\left(k_{1}, \ldots, k_{n}\right)$ and $\underline{x} \underline{\underline{k}}=x_{1}^{k_{1}} \cdot \ldots \cdot x_{n}^{k_{n}}$. The support of $f$ is $\operatorname{supp} f=\{\underline{k} \in$ $\left.\mathbb{Z}_{\geq 0}^{n} \mid c_{\underline{k}} \neq 0\right\}$. The Newton polyhedron $\Gamma_{0}$ of $f$ at the origin is the convex hull in $\mathbb{R}_{\geq 0}^{n}$ of

$$
\bigcup_{\underline{k} \in \operatorname{supp} f} \underline{k}+\mathbb{R}_{\geq 0}^{n}
$$

A facet of the Newton polyhedron is a face of dimension $n-1$. For a face $\tau$ of $\Gamma_{0}$, one defines the polynomial $f_{\tau}(\underline{x}):=\sum_{\underline{k} \in \mathbb{Z}^{n} \cap \tau} c_{\underline{k}} \underline{x} \underline{\underline{k}}$.

We say that the polynomial $f$ is nondegenerate over $\mathbb{C}$ w.r.t. the compact faces of $\Gamma_{0}$ (resp. nondegenerate over $\mathbb{C}$ w.r.t. the faces of $\Gamma_{0}$ ), if for every compact face $\tau$ (resp. for every face $\tau$ ) of $\Gamma_{0}$, the zero locus of $f_{\tau}$ has no singularities in $\left(\mathbb{C}^{\times}\right)^{n}$. For a fixed 
Newton polyhedron $\Gamma$, almost all polynomials having $\Gamma$ as their Newton polyhedron are nondegenerate w.r.t. the faces of $\Gamma$ (see [AVG, p.157]).

Let $K$ be a non-archimedean completion of $F$ with valuation ring $R$ and maximal ideal $P$, whose residue field we denote by $\mathbb{F}_{q}$. Note that $\mathcal{O}_{F} \subset R$, so it makes sense to consider $\bar{f}$, the polynomial over $\mathbb{F}_{q}$ obtained from $f$ by reducing each of its coefficients modulo $P$. We say that $\bar{f}$ is nondegenerate over $\mathbb{F}_{q}$ w.r.t. the compact faces of $\Gamma_{0}$ (resp. nondegenerate over $\mathbb{F}_{q}$ w.r.t. the faces of $\Gamma_{0}$ ) if for every compact face $\tau$ (resp. for every face $\tau$ ) of $\Gamma_{0}$, the zero locus of $\bar{f}_{\tau}$ has no singularities in $\left(\mathbb{F}_{q}^{\times}\right)^{n}$. If $f$ is nondegenerate over $\mathbb{C}$ w.r.t. the compact faces (resp. the faces) of its Newton polyhedron $\Gamma_{0}$, then recall that $\bar{f}$ is nondegenerate over $\mathbb{F}_{q}$ w.r.t. the compact faces (resp. the faces) of $\Gamma_{0}$ for almost all choices of $K$. Thus in order to prove the holomorphy conjecture for polynomials that are nondegenerate over $\mathbb{C}$, it suffices to restrict to completions $K$ for which moreover $\bar{f}$ is nondegenerate over the residue field $\mathbb{F}_{q}$.

Further on we will use the following property of nondegeneracy ([LVP2, Lemma 9]):

Lemma 2. If a complex polynomial $f(x, y, z)$ is nondegenerate w.r.t. the compact faces of its Newton polyhedron at the origin, then for almost all $k \in \mathbb{C}$ the polynomial $f(x, y, z-k)$ is nondegenerate w.r.t. the compact faces of its Newton polyhedron at the origin. (Analogously for the variables $x$ and $y$.)

\subsection{Some combinatorial data associated to the Newton polyhedron}

Let $\Gamma_{0}$ be as above. For $\underline{a}=\left(a_{1}, \ldots, a_{n}\right) \in \mathbb{R}_{\geq 0}^{n}$ we put

$$
N(\underline{a}):=\inf _{\underline{x} \in \Gamma_{0}} \underline{a} \cdot \underline{x}, \quad \nu(\underline{a}):=\sum_{i=1}^{n} a_{i}, \quad F(\underline{a}):=\left\{\underline{x} \in \Gamma_{0} \mid \underline{a} \cdot \underline{x}=N(\underline{a})\right\} .
$$

All $F(\underline{a}), \underline{a} \neq \underline{0}$, are faces of $\Gamma_{0}$. To a face $\tau$ of $\Gamma_{0}$ we associate its dual cone $\Delta_{\tau}=\{\underline{a} \in$ $\left.\mathbb{R}_{\geq 0}^{n} \mid F(\underline{a})=\tau\right\}$. It is a rational polyhedral cone of dimension $n-\operatorname{dim} \tau$. In particular if $\tau$ is a facet then $\Delta_{\tau}$ is a ray, say $\Delta_{\tau}=\underline{a} \mathbb{R}_{>0}$ for some non-zero $\underline{a} \in \mathbb{Z}_{\geq 0}^{n}$, and then the equation of the hyperplane through $\tau$ is $\underline{a} \cdot \underline{x}=N(\underline{a})$. If we demand that $\underline{a}$ is primitive, i.e. that $\operatorname{gcd}\left(a_{1}, \ldots, a_{n}\right)=1$, then this $\underline{a}$ is uniquely defined. For a facet $\tau$ we also use the notation $N(\tau)$ - called the lattice distance of $\tau$ - and $\nu(\tau)$, meaning respectively $N(\underline{a})$ and $\nu(\underline{a})$ for this associated $\underline{a} \in \mathbb{Z}_{\geq 0}^{n}$. For a general proper face $\tau$ the dual cone $\Delta_{\tau}$ is strictly positively spanned by the dual cones of the facets containing $\tau$.

For a set of linearly independent vectors $\underline{a_{1}}, \ldots, \underline{a_{r}} \in \mathbb{Z}^{n}$ we define the multiplicity $\operatorname{mult}\left(\underline{a_{1}}, \ldots, \underline{a_{r}}\right)$ as the index of the lattice $\mathbb{Z} \underline{a_{1}}+\ldots+\mathbb{Z} \underline{a_{r}}$ in the group of the points with integral coordinates of the subspace of $\overline{\mathbb{R}^{n}}$ generated by $\underline{a_{1}}, \ldots, \underline{a_{r}}$. Alternatively, $\operatorname{mult}\left(\underline{a_{1}}, \ldots, \underline{a_{r}}\right)$ is equal to the greatest common divisor of the determinants of the $(r \times r)$-matrices obtained by omitting columns from the matrix with rows $\underline{a_{1}}, \ldots, \underline{a_{r}}$. If $\Delta_{\tau}$ is a simplicial cone then by mult $\left(\Delta_{\tau}\right)$ we mean the multiplicity of its set of primitive generators. For a simplicial face $\tau$ we write $\operatorname{mult}(\tau)$ for the multiplicity of its set of vertices. 


\subsection{The Igusa zeta function with character for nondegenerate singu- larities}

In the case where $f \in R[\underline{x}]$ is nondegenerate over $\mathbb{F}_{q}$ w.r.t. the compact faces (resp. the faces) of its Newton polyhedron at the origin $\Gamma_{0}$, Hoornaert gave a formula $([\mathrm{H}$, Theorem 3.4]) for the local (resp. global) Igusa zeta function associated to $f$ and $\chi$ in terms of $\Gamma_{0}$, which we recall. Hoornaert states the formula for $R=\mathbb{Z}_{p}$ only, but her proof generalizes word by word to our more general setting.

Recall that we assume $\chi: R^{\times} \rightarrow \mathbb{C}^{\times}$to be non-trivial of conductor 1 . Let pr : $R^{\times} \rightarrow \mathbb{F}_{q}^{\times} \cong R^{\times} /(1+P)$ be the natural surjective homomorphism. As $\chi$ is trivial on $1+P$, there exists a unique homomorphism $\bar{\chi}: \mathbb{F}_{q}^{\times} \rightarrow \mathbb{C}^{\times}$such that $\chi=\bar{\chi} \circ \pi$. One formally puts $\bar{\chi}(0)=0$. Note that the order of $\chi$ divides the order of $\bar{\chi}$. Let $f$ be a non-zero polynomial over $R$ satisfying $f(\underline{0})=0$ and let $\bar{f}$ be nondegenerate over $\mathbb{F}_{q}$ w.r.t. all the compact faces (resp. all the faces) of its Newton polyhedron $\Gamma_{0}$. Let

$$
L_{\tau}:=q^{-n} \sum_{\underline{x} \in\left(\mathbb{F}_{q}^{\times}\right)^{n}} \bar{\chi}\left(\bar{f}_{\tau}(\underline{x})\right) \quad \text { and } \quad S\left(\Delta_{\tau}\right)(s):=\sum_{\underline{a} \in \mathbb{Z}^{n} \cap \Delta_{\tau}} q^{-\nu(\underline{a})-N(\underline{a}) s} .
$$

Then Hoornaert proved that the local, resp. global, Igusa zeta function associated to $f$ and the non-trivial character $\chi$ can be computed as

$$
Z_{f, 0}(\chi, s)=\sum_{\substack{\tau \text { compact } \\ \text { face of } \Gamma_{0}}} L_{\tau} S\left(\Delta_{\tau}\right)(s), \quad \text { resp. } \quad Z_{f}(\chi, s)=\sum_{\substack{\tau \\ \text { face of } \Gamma_{0}}} L_{\tau} S\left(\Delta_{\tau}\right)(s) .
$$

In the last summation also the face $\tau=\Gamma_{0}$ is included and $S\left(\Delta_{\Gamma_{0}}\right)=1$.

If $\Delta_{\tau}$ is simplicial, say (strictly positively) spanned by primitive linearly independent vectors $\underline{a}_{1}, \ldots, \underline{a}_{r} \in \mathbb{Z}_{\geq 0}^{n}$, then

$$
S\left(\Delta_{\tau}\right)(s)=\frac{\sum_{\underline{h}} q^{\nu(\underline{h})+N(\underline{h}) s}}{\prod_{i}\left(q^{\nu}\left(\underline{a}_{i}\right)+N\left(\underline{a}_{i}\right) s-1\right)}
$$

where the sum runs over $\mathbb{Z}^{n} \cap\left\{\lambda_{1} \underline{a}_{1}+\cdots+\lambda_{r} \underline{a}_{r} \mid 0 \leq \lambda_{i}<1\right\}$. In particular if $\operatorname{mult}\left(\Delta_{\tau}\right)=1$ then the numerator is 1 . In the non-simplicial case $S\left(\Delta_{\tau}\right)(s)$ is a sum of such expressions (obtained by subdividing $\Delta_{\tau}$ into simplicial cones).

We clearly see that the real parts of a set of candidate poles (containing all poles) of the local and global Igusa zeta function are given by the rational numbers $-\nu(\underline{a}) / N(\underline{a})$ for $\underline{a}$ orthogonal to a facet of the Newton polyhedron at the origin. Moreover we can restrict to the facets for which the order of $\bar{\chi}$ divides $N(\underline{a})$ : this follows from Lemma 7 below. A fortiori we can restrict to those for which the order of $\chi$ divides $N(\underline{a})$. We say that such a facet contributes a candidate pole to $Z_{f, 0}(\chi, s)\left(\operatorname{resp} . Z_{f}(\chi, s)\right)$.

We finally remark that if $f$ is nondegenerate over $\mathbb{C}$ w.r.t. the compact faces of $\Gamma_{0}$, then the couples $(\nu(\underline{a}), N(\underline{a}))$ are part of the numerical data $\left(\nu_{j}, N_{j}\right)$ associated to a very explicit (namely, toric) embedded resolution of $f^{-1}\{0\}$ over $F$, that was first described by Varchenko in $[\mathrm{Va}$. Thus the fact that we can restrict to the case where the order of $\chi$ divides $N(\underline{a})$ also follows from Igusa's seminal work. 


\subsection{The formula of Varchenko for the zeta function of monodromy of $f$ in the origin}

Let $f:\left(\mathbb{C}^{n}, 0\right) \rightarrow(\mathbb{C}, 0)$ be a germ of a holomorphic function. Let $\mathcal{F}$ be the Milnor fibre of the Milnor fibration at the origin associated with $f$ and write $h_{*}^{i}: H^{i}(\mathcal{F}, \mathbb{C}) \rightarrow$ $H^{i}(\mathcal{F}, \mathbb{C}), i \geq 0$, for the monodromy transformations.

The zeta function of monodromy at the origin associated to $f$ is

$$
\zeta_{f, 0}(t):=\prod_{i \geq 0}\left(\operatorname{det}\left(\mathrm{id}^{i}-t h_{*}^{i} ; H^{i}(\mathcal{F}, \mathbb{C})\right)\right)^{(-1)^{(i+1)}},
$$

where $i d^{i}$ is the identical transformation on $H^{i}(\mathcal{F}, \mathbb{C})$. One calls $\alpha$ an eigenvalue of monodromy of $f$ at the origin if $\alpha$ is an eigenvalue for some $h_{*}^{i}: H^{i}(\mathcal{F}, \mathbb{C}) \rightarrow H^{i}(\mathcal{F}, \mathbb{C})$. Denef proved that every eigenvalue of monodromy of $f$ is a zero or a pole of the zeta function of monodromy at some point of $\{f=0\}$ ([D3]). Varchenko gave in [Va a formula for $\zeta_{f, 0}$ in terms of $\Gamma_{0}$ if $f$ is nondegenerate w.r.t. the compact faces of its Newton polyhedron at the origin $\Gamma_{0}$. He defines a function $\zeta_{\tau}(t)$ for every compact face $\tau$ of $\Gamma_{0}$ for which there exists a subset $I \subset\{1, \ldots, n\}$ with $\# I=\operatorname{dim}(\tau)+1$ such that $\tau \subset L_{I}:=\left\{x \in \mathbb{R}^{n} \mid \forall i \notin I: x_{i}=0\right\}$. We will call such faces $V$-faces and we will denote the corresponding index set (resp. linear space) to a $\mathrm{V}$-face $\tau$ by $I_{\tau}$ (resp. $L_{I_{\tau}}$ ). If a $\mathrm{V}$-face is a simplex, then we will call it a $V$-simplex.

For a face $\tau$ of dimension 0 , we put $\operatorname{Vol}(\tau)=1$. For every other compact face $\tau$, $\operatorname{Vol}(\tau)$ is defined as the volume of $\tau$ for the volume form $\omega_{\tau}$. This is a volume form on $\operatorname{Aff}(\tau)$, the affine space spanned by $\tau$, such that the parallelepiped spanned by a lattice-basis of $\mathbb{Z}^{n} \cap \operatorname{Aff}(\tau)$ has volume 1 . The product $(\operatorname{dim} \tau) ! \operatorname{Vol}(\tau)$ is also called the normalized volume of the face $\tau$ and will be denoted by $\operatorname{NV}(\tau)$.

For a $\mathrm{V}$-face $\tau$, let $\sum_{i \in I_{\tau}} a_{i} x_{i}=N(\tau)$ be the equation of $\operatorname{Aff}(\tau)$ in $L_{I_{\tau}}$, where $N(\tau)$ and all $a_{i}$ (for $i \in I_{\tau}$ ) are positive integers and their greatest common divisor is equal to 1 . We put

$$
\zeta_{\tau}(t):=\left(1-t^{N(\tau)}\right)^{\mathrm{NV}(\tau)} .
$$

In Va] Varchenko showed that the zeta function of monodromy of $f$ in the origin is equal to

$$
\zeta_{f, 0}(t)=\prod \zeta_{\tau}(t)^{(-1)^{\operatorname{dim}(\tau)}},
$$

where the product runs over all $\mathrm{V}$-faces $\tau$ of $\Gamma_{0}$.

For a fixed facet $\tau$ of $\Gamma_{0}$, we say that a $\mathrm{V}$-face $\sigma$ in $\Gamma_{0}$ contributes w.r.t. $\tau$ if $e^{-2 \pi i \nu(\tau) / N(\tau)}$ is a zero of $\zeta_{\sigma}(t)$.

If $n=3$, the formula of Varchenko for the zeta function of monodromy in the origin has a specific form that we describe below. We first partition every compact facet in simplices, let us say without introducing new vertices. For such a $\operatorname{simplex} \tau$, we define the factor $F_{\tau}$ as in [LVP2]:

$$
F_{\tau}:=\zeta_{\tau} \prod_{\sigma} \zeta_{\sigma}^{-1} \prod_{p} \zeta_{p}
$$

where the first product runs over the 1-dimensional $\mathrm{V}$-faces $\sigma$ in $\tau$ and the second product runs over the 0 -dimensional $\mathrm{V}$-faces $p$ of $\tau$ that are intersection points of two 
1-dimensional V-faces in $\tau$. In [LVP2, Proposition 8] it is shown that $F_{\tau}$ is a polynomial. Following the formula of Varchenko, the zeta function of monodromy in the origin can be written as

$$
\zeta_{f, 0}(t)=\prod_{\tau} F_{\tau} \prod_{\sigma} \zeta_{\sigma}^{-1} \prod_{p} \zeta_{p}
$$

where the first product runs over all 2-dimensional simplices $\tau$ obtained after subdividing the compact facets and the other products run over 1-dimensional V-faces $\sigma$ and 0 -dimensional $\mathrm{V}$-faces $p$ for which $\zeta_{\sigma}$, respectively $\zeta_{p}$, was not used in any $F_{\tau}$.

\section{Preliminary results on the normalized volume}

When searching for eigenvalues of monodromy using the formula of Varchenko, one has to compare normalized volumes of compact faces in a facet. This is the motivation for this section. For two faces $\sigma$ and $\sigma^{\prime}$ in a simplicial facet $\tau$, we will denote the smallest face containing $\sigma$ and $\sigma^{\prime}$ by $\sigma+\sigma^{\prime}$.

Lemma 3. Let $\sigma$ and $\sigma^{\prime}$ be two non-disjoint $V$-faces in a simplicial facet $\tau$. Then $\sigma \cap \sigma^{\prime}$ and $\sigma+\sigma^{\prime}$ are also $V$-faces.

Proof. Let $\sigma$ be a $d_{1}$-dimensional V-simplex and $\sigma^{\prime}$ a $d_{2}$-dimensional V-simplex, having $k$ vertices in common. Suppose that the vertices of $\sigma+\sigma^{\prime}$ have exactly $s$ zero entries in common. Then one has

$$
s \leq n-\#\left(\sigma+\sigma^{\prime}\right)=n-\left(d_{1}+1+d_{2}+1-k\right)
$$

where (abusing notation) $\#\left(\sigma+\sigma^{\prime}\right)$ denotes the number of vertices of $\sigma+\sigma^{\prime}$. On the other hand, the vertices of $\sigma \cap \sigma^{\prime}$ have at most $n-k$ zero entries in common, and so

$$
n-k \geq\left(n-d_{1}-1\right)+\left(n-d_{2}-1\right)-s
$$

Combining these two inequalities, one finds that they are actually equalities and so $\sigma \cap \sigma^{\prime}$ and $\sigma+\sigma^{\prime}$ are $\mathrm{V}$-simplices.

Recall that for a V-simplex $\tau$, the normalized volume $\mathrm{NV}(\tau)$ is equal to its multiplicity mult $(\tau)$ divided by its lattice distance $N(\tau)$. Let $B^{j}\left(B_{1}^{j}, \ldots, B_{n}^{j}\right), 1 \leq j \leq n$, be the vertices of $\tau$ and let $\sigma$ be a $\mathrm{V}$-face in $\tau$ with vertices $B^{1}, \ldots, B^{k}$ and $I_{\sigma}=\{1, \ldots, k\}$. Then $\operatorname{mult}(\tau)$ is the absolute value of the determinant of the matrix

$$
\left(\begin{array}{cccccc}
B_{1}^{1} & \ldots & B_{k}^{1} & 0 & \ldots & 0 \\
\vdots & \ldots & \vdots & \vdots & \ldots & \vdots \\
B_{1}^{k} & \ldots & B_{k}^{k} & 0 & \ldots & 0 \\
* & \ldots & * & B_{k+1}^{k+1} & \ldots & B_{n}^{k+1} \\
\vdots & \ldots & \vdots & \vdots & \ldots & \vdots \\
* & \ldots & * & B_{k+1}^{n} & \ldots & B_{n}^{n}
\end{array}\right)
$$


We will denote the matrix

$$
M_{\tau, \sigma}:=\left(\begin{array}{ccc}
B_{k+1}^{k+1} & \ldots & B_{n}^{k+1} \\
\vdots & \ldots & \vdots \\
B_{k+1}^{n} & \ldots & B_{n}^{n}
\end{array}\right) .
$$

Then we have that $\operatorname{mult}(\tau)=\operatorname{mult}(\sigma)\left|\operatorname{det}\left(M_{\tau, \sigma}\right)\right|$.

Proposition 4. Let $\tau$ be a simplicial facet of a Newton polyhedron in $\mathbb{R}^{n}$. If $\sigma$ is a $V$-face in $\tau$, then $\mathrm{NV}(\sigma) \mid \mathrm{NV}(\tau)$.

Proof. Let us denote the equation of the affine space through $\tau$ resp. through $\sigma$ by

$$
\operatorname{Aff}(\tau) \leftrightarrow a_{1} x_{1}+\ldots+a_{n} x_{n}=N(\tau), \quad \operatorname{Aff}(\sigma) \leftrightarrow \frac{a_{1} x_{1}+\ldots+a_{k} x_{k}}{\operatorname{gcd}\left(a_{1}, \ldots, a_{k}\right)}=N(\sigma),
$$

with $\operatorname{gcd}\left(a_{1}, \ldots, a_{n}\right)=1$ and $N(\sigma)=N(\tau) / \operatorname{gcd}\left(a_{1}, \ldots, a_{k}\right)$. Let $B^{j}\left(B_{1}^{j}, \ldots, B_{n}^{j}\right), 1 \leq$ $j \leq n$, be the vertices of $\tau$ and $B^{k+1}, \ldots, B^{n}$ the vertices of $\tau$ that are not contained in $\sigma$. Then we find that

$$
\mathrm{NV}(\tau)=\frac{\operatorname{NV}(\sigma)\left|\operatorname{det}\left(M_{\tau, \sigma}\right)\right|}{\operatorname{gcd}\left(a_{1}, \ldots, a_{k}\right)}
$$

Let $v_{j}\left(B_{j}^{k+1}, \ldots, B_{j}^{n}\right)^{T}, k+1 \leq j \leq n$, be the $j$ th column of the matrix $M_{\tau, \sigma}$ and let $\tilde{M_{\tau, \sigma}}$ be the matrix obtained from $M_{\tau, \sigma}$ by replacing the first column by $a_{k+1} v_{k+1}+\ldots+a_{n} v_{n}$. For every vertex $B^{j}$ of $\tau$ we have that $\operatorname{gcd}\left(a_{1}, \ldots, a_{k}\right) \mid a_{k+1} B_{k+1}^{j}+\ldots+a_{n} B_{n}^{j}$ and hence we find that $\operatorname{gcd}\left(a_{1}, \ldots, a_{k}\right) \mid \operatorname{det}\left(\tilde{M_{\tau, \sigma}}\right)=a_{k+1} \operatorname{det}\left(M_{\tau, \sigma}\right)$. Analogously, we obtain that $\operatorname{gcd}\left(a_{1}, \ldots, a_{k}\right) \mid a_{j} \operatorname{det}\left(M_{\tau, \sigma}\right)$, for $k+1 \leq j \leq n$. As we supposed that $\operatorname{gcd}\left(a_{1}, \ldots, a_{n}\right)=$ 1 , we get that $\operatorname{gcd}\left(a_{1}, \ldots, a_{k}\right) \mid \operatorname{det}\left(M_{\tau, \sigma}\right)$, which implies that $\mathrm{NV}(\sigma) \mid \mathrm{NV}(\tau)$.

Proposition 5. Let $\tau$ be a simplicial facet of a Newton polyhedron in $\mathbb{R}^{n}$. If $\sigma$ and $\sigma^{\prime}$ are $V$-faces in $\tau$ such that $\sigma \cap \sigma^{\prime} \neq \emptyset$, then

$$
\mathrm{NV}(\tau) \mathrm{NV}\left(\sigma \cap \sigma^{\prime}\right)=\mathrm{NV}(\sigma) \mathrm{NV}\left(\sigma^{\prime}\right) M, \text { for some } M \in \mathbb{N} \text {. }
$$

Moreover, if $\sigma+\sigma^{\prime}=\tau$, then $M=1$ if and only if $N\left(\sigma \cap \sigma^{\prime}\right)=\operatorname{gcd}\left(N(\sigma), N\left(\sigma^{\prime}\right)\right)$.

Proof. As $\sigma+\sigma^{\prime}$ is also a V-face (see Lemma 3), it follows by Proposition 4 that it is sufficient to prove that

$$
\mathrm{NV}\left(\sigma+\sigma^{\prime}\right) \mathrm{NV}\left(\sigma \cap \sigma^{\prime}\right)=\mathrm{NV}(\sigma) \mathrm{NV}\left(\sigma^{\prime}\right) M, \quad \text { for some } M \in \mathbb{N} .
$$

Let $B^{1}, \ldots, B^{k}, B^{k+1}, \ldots, B^{r}$ be the vertices of $\sigma$ and $B^{1}, \ldots, B^{k}, B^{r+1}, \ldots, B^{s}$ be the vertices of $\sigma^{\prime}$. Then mult $\left(\sigma+\sigma^{\prime}\right)$ is the absolute value of the determinant of the matrix

$$
\left(\begin{array}{ccccccccc}
B_{1}^{1} & \ldots & B_{k}^{1} & 0 & \ldots & 0 & 0 & \ldots & 0 \\
\vdots & \ldots & \vdots & \vdots & \ldots & \vdots & \vdots & \ldots & \vdots \\
B_{1}^{k} & \ldots & B_{k}^{k} & 0 & \ldots & 0 & 0 & \ldots & 0 \\
* & \ldots & * & B_{k+1}^{k+1} & \ldots & B_{r}^{k+1} & 0 & \ldots & 0 \\
\vdots & \ldots & \vdots & \vdots & \ldots & \vdots & \vdots & \ldots & \vdots \\
* & \ldots & * & B_{k+1}^{r} & \ldots & B_{r}^{r} & 0 & \ldots & 0 \\
* & \ldots & * & 0 & \ldots & 0 & B_{r+1}^{r+1} & \ldots & B_{s}^{r+1} \\
\vdots & \ldots & \vdots & \vdots & \ldots & \vdots & \vdots & \ldots & \vdots \\
* & \ldots & * & 0 & \ldots & 0 & B_{r+1}^{s} & \ldots & B_{s}^{s}
\end{array}\right) .
$$


We write

$$
\begin{array}{r}
\operatorname{Aff}\left(\sigma+\sigma^{\prime}\right) \leftrightarrow a_{1} x_{1}+\ldots+a_{s} x_{s}=N\left(\sigma+\sigma^{\prime}\right), \quad \text { with } \operatorname{gcd}\left(a_{1}, \ldots, a_{s}\right)=1, \\
\alpha:=\operatorname{gcd}\left(a_{1}, \ldots, a_{k}\right), \beta:=\operatorname{gcd}\left(a_{k+1}, \ldots, a_{r}\right) \text { and } \gamma:=\operatorname{gcd}\left(a_{r+1}, \ldots, a_{s}\right) .
\end{array}
$$

Then we have

$$
\begin{aligned}
\operatorname{Aff}(\sigma) & \leftrightarrow \frac{a_{1} x_{1}+\ldots+a_{k} x_{k}+a_{k+1} x_{k+1}+\ldots+a_{r} x_{r}}{\operatorname{gcd}(\alpha, \beta)}=\frac{N\left(\sigma+\sigma^{\prime}\right)}{\operatorname{gcd}(\alpha, \beta)}=N(\sigma), \\
\operatorname{Aff}\left(\sigma^{\prime}\right) & \leftrightarrow \frac{a_{1} x_{1}+\ldots+a_{k} x_{k}+a_{r+1} x_{r+1}+\ldots+a_{s} x_{s}}{\operatorname{gcd}(\alpha, \gamma)}=\frac{N\left(\sigma+\sigma^{\prime}\right)}{\operatorname{gcd}(\alpha, \gamma)}=N\left(\sigma^{\prime}\right), \\
\operatorname{Aff}\left(\sigma \cap \sigma^{\prime}\right) & \leftrightarrow \frac{a_{1} x_{1}+\ldots+a_{k} x_{k}}{\alpha}=\frac{N\left(\sigma+\sigma^{\prime}\right)}{\alpha}=N\left(\sigma \cap \sigma^{\prime}\right) .
\end{aligned}
$$

By using Proposition 4 we get

$$
\mathrm{NV}\left(\sigma+\sigma^{\prime}\right) \operatorname{NV}\left(\sigma \cap \sigma^{\prime}\right)=\mathrm{NV}(\sigma) \operatorname{NV}\left(\sigma^{\prime}\right) \frac{\alpha}{\operatorname{gcd}(\alpha, \beta) \operatorname{gcd}(\alpha, \gamma)} .
$$

As $\operatorname{gcd}(\alpha, \beta, \gamma)=1$, the quotient $\alpha /(\operatorname{gcd}(\alpha, \beta) \operatorname{gcd}(\alpha, \gamma))$ is an integer.

To prove the second statement, let $\sigma$ and $\sigma^{\prime}$ be two $\mathrm{V}$-faces in a simplicial facet $\tau$ such that $\sigma+\sigma^{\prime}=\tau$. Then one easily shows that $N(\tau)=\operatorname{lcm}\left(N(\sigma), N\left(\sigma^{\prime}\right)\right)$ and one can then write

$$
M=\frac{\alpha}{\operatorname{gcd}(\alpha, \beta) \operatorname{gcd}(\alpha, \gamma)}=\frac{\operatorname{gcd}\left(N(\sigma), N\left(\sigma^{\prime}\right)\right)}{N\left(\sigma \cap \sigma^{\prime}\right)} .
$$

Corollary 6. Let $\sigma$ and $\sigma^{\prime}$ be two $V$-faces in a simplicial facet $\tau$. If $\sigma$ and $\sigma^{\prime}$ contribute w.r.t. $\tau$ and if $\sigma \cap \sigma^{\prime}$ does not, then $M \geq 2$ in Equation (4).

\section{Some character sums}

In order to prove the holomorphy conjecture, we will have to show that some candidate poles of $Z_{f, 0}(\chi, s)$ (resp. $\left.Z_{f}(\chi, s)\right)$ are false poles. These proofs rely on the computation of some character sums. We first recall some well-known properties of character sums over finite fields which we will need when treating $B_{1}$-facets. We then study a specific character sum (see Proposition 10) which shows up when proving fakeness of some other candidate pole.

Lemma 7. Let $a_{1}, \ldots, a_{n}, N \in \mathbb{Z}$ and let $\chi$ be a multiplicative character of $\mathbb{F}_{q}^{\times}$whose order is not a divisor of $N$. Let $f \in \mathbb{F}_{q}\left[x_{1}, \ldots, x_{n}\right]$ be such that each exponent $\left(k_{1}, \ldots, k_{n}\right)$ appearing in $f$ satisfies $a_{1} k_{1}+\cdots+a_{n} k_{n}=N$. Then

$$
\sum_{\left(x_{1}, \ldots, x_{n}\right) \in\left(\mathbb{F}_{q}^{\times}\right)^{n}} \chi\left(f\left(x_{1}, \ldots, x_{n}\right)\right)=0 .
$$

Proof. Pick $u \in \mathbb{F}_{q}^{\times}$such that $\chi\left(u^{N}\right) \neq 1$. Then the left hand side equals

$$
\sum_{\left(x_{1}, \ldots, x_{n}\right) \in\left(\mathbb{F}_{q}^{\times}\right)^{n}} \chi\left(f\left(u^{a_{1}} x_{1}, \ldots, u^{a_{n}} x_{n}\right)\right)=\chi\left(u^{N}\right) \sum_{\left(x_{1}, \ldots, x_{n}\right) \in\left(\mathbb{F}_{q}^{\times}\right)^{n}} \chi\left(f\left(x_{1}, \ldots, x_{n}\right)\right)
$$

from which the property follows. 
Lemma 8. Let $a \in \mathbb{N}$ and let $\chi$ be a multiplicative character of $\mathbb{F}_{q}^{\times}$whose order is not a divisor of $a$, then $\sum_{x \in \mathbb{F}_{q}^{\times}} \chi\left(x^{a}\right)=0$.

Proof. Take $f(x)=x^{a}$ in the foregoing lemma.

Lemma 9. Let $f$ be a polynomial and $g$ be a monomial (possibly equipped with a non-zero coefficient) over $\mathbb{F}_{q}$ in the variables $x_{2}, \ldots, x_{n}$, and let $\chi$ be a non-trivial multiplicative character of $\mathbb{F}_{q}^{\times}$. Then

$\sum_{\left(x_{1}, \ldots, x_{n}\right) \in\left(\mathbb{F}_{q}^{\times}\right)^{n}} \chi\left(f\left(x_{2}, \ldots, x_{n}\right)+x_{1} g\left(x_{2}, \ldots, x_{n}\right)\right)=-\sum_{\left(x_{2}, \ldots, x_{n}\right) \in\left(\mathbb{F}_{q}^{\times}\right)^{n-1}} \chi\left(f\left(x_{2}, \ldots, x_{n}\right)\right)$.

Proof. One can write

$$
\begin{aligned}
& \sum_{\left(x_{1}, \ldots, x_{n}\right) \in\left(\mathbb{F}_{q}^{\times}\right)^{n}} \chi\left(f\left(x_{2}, \ldots, x_{n}\right)+x_{1} g\left(x_{2}, \ldots, x_{n}\right)\right) \\
= & \sum_{\left(x_{2}, \ldots, x_{n}\right) \in\left(\mathbb{F}_{q}^{\times}\right)^{n-1}} \sum_{x_{1} \in \mathbb{F}_{q}^{\times}} \chi\left(f\left(x_{2}, \ldots, x_{n}\right)+x_{1} g\left(x_{2}, \ldots, x_{n}\right)\right) \\
= & \sum_{\left(x_{2}, \ldots, x_{n}\right) \in\left(\mathbb{F}_{q}^{\times}\right)^{n-1}}\left(\sum_{u \in \mathbb{F}_{q}} \chi(u)-\chi\left(f\left(x_{2}, \ldots, x_{n}\right)\right)\right) \\
= & -\sum_{\left(x_{2}, \ldots, x_{n}\right) \in\left(\mathbb{F}_{q}^{\times}\right)^{n-1}} \chi\left(f\left(x_{2}, \ldots, x_{n}\right)\right),
\end{aligned}
$$

where we used Lemma 8 in the last step.

Proposition 10. Let $\chi$ be a multiplicative character of $\mathbb{F}_{q}^{\times}$such that its order does not divide $a \in \mathbb{N}$. Let $\alpha \in \mathbb{F}_{q}$ and $\beta, \gamma \in \mathbb{F}_{q}^{\times}$. Then

$$
\sum_{x, y, z \in \mathbb{F}_{q}^{\times}} \chi\left(\alpha x^{a}+\beta x^{i} y^{2}+\gamma x^{i} z^{2}\right)=-\sum_{x, y \in \mathbb{F}_{q}^{\times}} \chi\left(\alpha x^{a}+\beta x^{i} y^{2}\right)-\sum_{x, z \in \mathbb{F}_{q}^{\times}} \chi\left(\alpha x^{a}+\gamma x^{i} z^{2}\right),
$$

with $i \in \mathbb{N}$.

Proof. First notice that we can reduce to the cases $i=0$ or $i=1$. We can also assume that $q$ is odd: indeed if $q$ is even then we can replace $y^{2}$ by $y$ and $z^{2}$ by $z$, from which one sees that all sums are zero, for instance by using Lemma 8 and Lemma 9 . Let

$$
\varepsilon= \begin{cases}2 & \text { if }-\beta / \gamma \text { is a square in } \mathbb{F}_{q}, \\ 0 & \text { if not. }\end{cases}
$$

If $i=0$, then for each $c \in \mathbb{F}_{q}^{\times}$define

$$
\begin{gathered}
L_{c}:=\#\left\{(x, y, z) \in \mathbb{F}_{q}^{\times 3} \mid \alpha x^{a}+\beta y^{2}+\gamma z^{2}=c\right\}, \quad M_{c}:=\#\left\{x \in \mathbb{F}_{q}^{\times} \mid \alpha x^{a}=c\right\}, \\
N_{1, c}:=\#\left\{(x, y) \in \mathbb{F}_{q}^{\times 2} \mid \alpha x^{a}+\beta y^{2}=c\right\}, \quad N_{2, c}:=\#\left\{(x, z) \in \mathbb{F}_{q}^{\times 2} \mid \alpha x^{a}+\gamma z^{2}=c\right\} .
\end{gathered}
$$

We rewrite the first equation as

$$
\beta y^{2}+\gamma z^{2}=c-\alpha x^{a} .
$$


For each value of $x \in \mathbb{F}_{q}^{\times}$this defines a conic in the variables $y$ and $z$. In the $M_{c}$ cases where $c-\alpha x^{a}=0$ the conic carries $\varepsilon(q-1)+1$ points $(y, z) \in \mathbb{F}_{q}^{2}$. If $c-\alpha x^{a} \neq 0$ then Equation (5) defines a smooth conic having $\varepsilon$ points at infinity. As every projective nonsingular curve of genus 0 over a finite field $\mathbb{F}_{q}$ has always $q+1$ points (see $[\mathrm{W}]$ ), the conic carries $q+1-\varepsilon$ points in $\mathbb{F}_{q}^{2}$. We conclude that there are

$$
(\varepsilon(q-1)+1) M_{c}+(q+1-\varepsilon)\left(q-1-M_{c}\right)
$$

solutions $(x, y, z) \in \mathbb{F}_{q}^{\times} \times \mathbb{F}_{q}^{2}$ to Equation (50). Because in $L_{c}$ there are $M_{c}$ points of the form $(x, 0,0), N_{1, c}$ points of the form $(x, y, 0)$ with $y \neq 0$, and $N_{2, c}$ points of the form $(x, 0, z)$ with $z \neq 0$, we conclude that

$$
L_{c}=(\varepsilon(q-1)+1) M_{c}+(q+1-\varepsilon)\left(q-1-M_{c}\right)-M_{c}-N_{1, c}-N_{2, c} .
$$

Then for some constant $\lambda$, it holds that $L_{c}=-N_{1, c}-N_{2, c}+\lambda M_{c}$. Now note that

$$
\begin{gathered}
S_{1}:=\sum_{x, y, z \in \mathbb{F}_{q}^{\times}} \chi\left(\alpha x^{a}+\beta y^{2}+\gamma z^{2}\right)=\sum_{c \in \mathbb{F}_{q}^{\times}} L_{c} \chi(c), \\
S_{2}:=\sum_{x, y \in \mathbb{F}_{q}^{\times}} \chi\left(\alpha x^{a}+\beta y^{2}\right)=\sum_{c \in \mathbb{F}_{q}^{\times}} N_{1, c} \chi(c), \quad S_{3}:=\sum_{x, z \in \mathbb{F}_{q}^{\times}} \chi\left(\alpha x^{a}+\gamma z^{2}\right)=\sum_{c \in \mathbb{F}_{q}^{\times}} N_{2, c} \chi(c), \\
0=\chi(\alpha) \sum_{x \in \mathbb{F}_{q}^{\times}} \chi\left(x^{a}\right)=\sum_{x \in \mathbb{F}_{q}^{\times}} \chi\left(\alpha x^{a}\right)=\sum_{c \in \mathbb{F}_{q}^{\times}} M_{c} \chi(c) .
\end{gathered}
$$

In the last case, the first equality follows by Lemma 8 , Plugging in the expression for $L_{c}$ in $S_{1}$ we find

$$
S_{1}=-\sum_{c \in \mathbb{F}_{q}^{\times}} N_{1, c} \chi(c)-\sum_{c \in \mathbb{F}_{q}^{\times}} N_{2, c} \chi(c)+\lambda \sum_{c \in \mathbb{F}_{q}^{\times}} N_{c} \chi(c)=-S_{2}-S_{3}
$$

If $i=1$, one instead of (5) uses the conic

$$
\beta y^{2}+\gamma z^{2}=\frac{c-\alpha x^{a}}{x}
$$

and proceeds analogously.

Note that the exponents $(a, 0,0),(i, 2,0),(i, 0,2)$ are contained in the hyperplane $2 k_{1}+(a-i) k_{2}+(a-i) k_{3}=2 a$, so under the stronger assumption that the order of $\chi$ does not divide $2 a$, or under the additional assumption that $a-i$ is even, we see from Lemma 7 that all sums in the statement of the proposition are actually zero.

\section{A proof of the holomorphy conjecture for nondegener- ate surface singularities}

Let $f(\underline{x})$ be as in Subsection 2.1 and assume that it is nondegenerate over $\mathbb{C}$ with respect to the compact faces (resp. the faces) of its Newton polyhedron at the origin 
$\Gamma_{0}$. Let $K$ be a non-archimedean completion with valuation ring $R$ and residue field $\mathbb{F}_{q}$, such that $\bar{f}$ is nondegenerate over $\mathbb{F}_{q}$ with respect to the compact faces (resp. the faces) of $\Gamma_{0}$. Let $\chi: R^{\times} \rightarrow \mathbb{C}^{\times}$be a non-trivial character of conductor 1 . If $Z_{f, 0}(\chi, s)$ (resp. $\left.Z_{f}(\chi, s)\right)$ is not holomorphic on $\mathbb{C}$, then by the material from Subsection 2.3 it has a pole with real part equal to $-\nu(\tau) / N(\tau)$ for some facet $\tau$ of $\Gamma_{0}$ for which the order of $\bar{\chi}$ divides $N(\tau)$. Here as before $\bar{\chi}$ denotes the unique character of $\mathbb{F}_{q}^{\times}$associated to $\chi$.

For some facets $\tau$, in particular the $B_{1}$-facets and the $X_{2}$-facets which we introduce here, we will mostly have to prove that $-\nu(\tau) / N(\tau)$ cannot be the real part of a pole of $Z_{f, 0}(\chi, s)$ (resp. $Z_{f}(\chi, s)$ ). For the other facets, we will prove that $e^{-2 \pi i / N(\tau)}$ is an eigenvalue of monodromy of $f$ at some point of $f^{-1}\{0\}$ and we will thus obtain that the order of $\chi$ (which we recall divides the order of $\bar{\chi}$ ) divides the order of some eigenvalue of monodromy at some point of $f^{-1}\{0\}$.

Let us first recall the notion of $B_{1}$-facets, introduced in [LVP2]. A simplicial facet of an $n$-dimensional Newton polyhedron $(n \geq 2)$ is a $B_{1}$-simplex w.r.t. the variable $x_{i}$ if it is a simplex with $n-1$ vertices in the coordinate hyperplane $x_{i}=0$ and one vertex at distance one of this hyperplane. We say that a facet $\tau$ of an $n$-dimensional Newton polyhedron is non-compact for the variable $x_{j}(1 \leq j \leq n)$ if for every point $p \in \tau$ the point $p+(0, \ldots, 0,1,0, \ldots, 0) \in \tau$, where $(0, \ldots, 0,1,0, \ldots, 0)$ is an $n$-tuple with 1 at place $j$ and 0 everywhere else. We define the maps $\pi_{j}: \mathbb{R}^{n} \rightarrow \mathbb{R}^{n-1}$ : $\left(x_{1}, \ldots, x_{n}\right) \mapsto\left(x_{1}, \ldots, \widehat{x_{j}}, \ldots, x_{n}\right)$ for $j=1, \ldots, n$. A non-compact facet $\tau$ of an $n$ dimensional Newton polyhedron $(n \geq 3)$ is a (non-compact) $B_{1}$-facet w.r.t. the variable $x_{i}$ if $\tau$ is non-compact for exactly one variable $x_{j}$ and if $\pi_{j}(\tau)$ is a $B_{1}$-simplex in $\mathbb{R}^{n-1}$ w.r.t. $x_{i}$. A $B_{1}$-facet is a $B_{1}$-simplex or a non-compact $B_{1}$-facet w.r.t. some variable.

Definition 11. A facet of type $X_{2}$ in a 3-dimensional Newton polyhedron is a facet whose vertices (up to permutation of the coordinates) are of the form $p(a, 0,0), q\left(x_{1}, 0,2\right)$, $r\left(x_{2}, 2,0\right)$ with $a-x_{2}$ and $a-x_{1}$ both odd.

\subsection{Determination of a set of eigenvalues}

In [LVP2, Theorem 10] Van Proeyen and the third author proved that $e^{-2 \pi i \nu(\tau) / N(\tau)}$ is an eigenvalue of monodromy of $f$ at some point of $f^{-1}\{0\}$, whenever $\tau$ is not a $B_{1^{-}}$ facet. We will now show that $e^{-2 \pi i / N(\tau)}$ is also an eigenvalue of monodromy at some point of $f^{-1}\{0\}$, except for some cases which will be treated in Subsection 5.2. We here rely on Proposition 5 to get a more conceptual proof.

We first divide the compact facets in simplices $\tau$, without introducing new vertices.

Proposition 12. If $\tau$ is not of type $B_{1}$ nor of type $X_{2}$, then $e^{-2 \pi i / N(\tau)}$ is a zero of $F_{\tau}$.

Proof. Case 1: $\tau$ does not Contain a Segment in a Coordinate Plane.

By formula (2),$F_{\tau}=\zeta_{\tau}=\left(1-t^{N(\tau)}\right)^{\mathrm{NV}(\tau)}$ and $e^{-2 \pi i / N(\tau)}$ clearly is a zero of $F_{\tau}$.

Case 2: $\tau$ Contains exactly one 1-Dimensional V-face $\sigma$.

In this case, we have

$$
F_{\tau}=\frac{\zeta_{\tau}}{\zeta_{\sigma}}=\frac{\left(1-t^{N(\tau)}\right)^{\mathrm{NV}(\tau)}}{\left(1-t^{N(\sigma)}\right)^{\mathrm{NV}(\sigma)}}
$$


Then $e^{-2 \pi i / N(\tau)}$ is a zero of $F_{\tau}$ unless $N(\sigma)=N(\tau)$ and $\operatorname{NV}(\sigma)=\operatorname{NV}(\tau)$. One easily checks that then $\tau$ would be a $B_{1}$-facet.

Case 3: $\tau$ COntains exactly two 1-Dimensional V-Faces $\sigma_{1}$ And $\sigma_{2}$. In this situation,

$$
F_{\tau}=\frac{\zeta_{\tau} \zeta_{p}}{\zeta_{\sigma_{1}} \zeta_{\sigma_{2}}}=\frac{\left(1-t^{l}\right)\left(1-t^{N(\tau)}\right)^{\mathrm{NV}(\tau)}}{\left(1-t^{N\left(\sigma_{1}\right)}\right)^{\mathrm{NV}\left(\sigma_{1}\right)}\left(1-t^{N\left(\sigma_{2}\right)}\right)^{\mathrm{NV}\left(\sigma_{2}\right)}}
$$

where w.l.o.g. $\{p(l, 0,0)\}=\sigma_{1} \cap \sigma_{2}$.

If $N\left(\sigma_{1}\right) \neq N(\tau)$ or $N\left(\sigma_{2}\right) \neq N(\tau)$, then see Case 1 and Case 2. If $N(\tau)=N\left(\sigma_{1}\right)=$ $N\left(\sigma_{2}\right)$, then $F_{\tau}=\left(1-t^{l}\right)\left(1-t^{N(\tau)}\right)^{\mathrm{NV}(\tau)-\mathrm{NV}\left(\sigma_{1}\right)-\mathrm{NV}\left(\sigma_{2}\right)}$.

Case 3.1: If $N(p)=N(\tau)$, then by Proposition 15, $\mathrm{NV}(\tau)=\mathrm{NV}\left(\sigma_{1}\right) \mathrm{NV}\left(\sigma_{2}\right)$ and hence $F_{\tau}=\left(1-t^{N(\tau)}\right)^{\left(\mathrm{NV}\left(\sigma_{1}\right)-1\right)\left(\mathrm{NV}\left(\sigma_{2}\right)-1\right)}$. If $\mathrm{NV}\left(\sigma_{1}\right)$ or $\mathrm{NV}\left(\sigma_{2}\right)$ would be equal to 1 , then it would result that $\mathrm{NV}(\tau)=\mathrm{NV}\left(\sigma_{i}\right)$, for some $i \in\{1,2\}$ and again $\tau$ would be a $B_{1}$-facet. Consequently, $e^{-2 \pi i / N(\tau)}$ is a zero of $F_{\tau}$.

Case 3.2: Suppose that $N(p) \neq N(\tau)$. By Proposition [5, we have $N V(\tau)=$ $M \operatorname{NV}\left(\sigma_{1}\right) \mathrm{NV}\left(\sigma_{2}\right)$, with $M \geq 2$. If not both $\mathrm{NV}\left(\sigma_{1}\right)$ and $\mathrm{NV}\left(\sigma_{2}\right)$ are equal to 1 , then one easily deduces that $\mathrm{NV}(\tau)-\mathrm{NV}\left(\sigma_{1}\right)-\mathrm{NV}\left(\sigma_{2}\right)>0$. If $\mathrm{NV}\left(\sigma_{1}\right)=\mathrm{NV}\left(\sigma_{2}\right)=1$, then $\mathrm{NV}(\tau)-\mathrm{NV}\left(\sigma_{1}\right)-\mathrm{NV}\left(\sigma_{2}\right)>0$ if and only if $M>2$. It remains thus to study the case $\mathrm{NV}(\tau)=M=2, \mathrm{NV}\left(\sigma_{1}\right)=\mathrm{NV}\left(\sigma_{2}\right)=1$. As we supposed that $N(\tau)=N\left(\sigma_{1}\right)=N\left(\sigma_{2}\right)$, the vertices of $\tau$ are then $p(N(\tau) / 2,0,0), q\left(x_{1}, 0,2\right), r\left(x_{2}, 2,0\right)$, and

$$
\operatorname{Aff}(\tau) \leftrightarrow 2 x+\left(N(\tau) / 2-x_{2}\right) y+\left(N(\tau) / 2-x_{1}\right) z=N(\tau)
$$

From $N\left(\sigma_{1}\right)=N\left(\sigma_{2}\right)=N(\tau)$ it follows that $N(\tau) / 2-x_{2}$ and $N(\tau) / 2-x_{1}$ are odd and hence $\tau$ is of type $X_{2}$.

Case 4: $\tau$ COntains three 1-Dimensional V-Faces $\sigma_{1}, \sigma_{2}$ And $\sigma_{3}$.

In this situation

$$
F_{\tau}=\frac{\zeta_{\tau} \zeta_{p} \zeta_{q} \zeta_{r}}{\zeta_{\sigma_{1}} \zeta_{\sigma_{2}} \zeta_{\sigma_{3}}}
$$

with $p=\sigma_{1} \cap \sigma_{2}, q=\sigma_{1} \cap \sigma_{3}$ and $r=\sigma_{2} \cap \sigma_{3}$. We suppose that $N(\tau)=N\left(\sigma_{1}\right)=$ $N\left(\sigma_{2}\right)=N\left(\sigma_{3}\right)$, if not then we fall back on one of the previous cases.

Case 4.1: If $N(\tau)=N\left(\sigma_{1} \cap \sigma_{2}\right)=N\left(\sigma_{1} \cap \sigma_{3}\right)=N\left(\sigma_{2} \cap \sigma_{3}\right)$, then, by Proposition 5. $\operatorname{NV}(\tau)=\mathrm{NV}\left(\sigma_{1}\right) \mathrm{NV}\left(\sigma_{2}\right)=\mathrm{NV}\left(\sigma_{1}\right) \mathrm{NV}\left(\sigma_{3}\right)=\mathrm{NV}\left(\sigma_{2}\right) \mathrm{NV}\left(\sigma_{3}\right)$, and thus $\mathrm{NV}\left(\sigma_{1}\right)=$ $\mathrm{NV}\left(\sigma_{2}\right)=\mathrm{NV}\left(\sigma_{3}\right)$. Then $F_{\tau}$ becomes

$$
F_{\tau}=\frac{\left(1-t^{N(\tau)}\right)^{\mathrm{NV}\left(\sigma_{1}\right)^{2}+3}}{\left(1-t^{N(\tau)}\right)^{3 \mathrm{NV}\left(\sigma_{1}\right)}} .
$$

Since $\operatorname{NV}\left(\sigma_{1}\right)^{2}+3>3 \mathrm{NV}\left(\sigma_{1}\right)$, it follows that $e^{-\frac{2 \pi i}{N(\tau)}}$ is a zero of $F_{\tau}$. 
Case 4.2: If $N(\tau)=N\left(\sigma_{1} \cap \sigma_{2}\right)=N\left(\sigma_{2} \cap \sigma_{3}\right) \neq N\left(\sigma_{1} \cap \sigma_{3}\right)$, then Proposition 5 yields $\mathrm{NV}(\tau)=\mathrm{NV}\left(\sigma_{1}\right) \mathrm{NV}\left(\sigma_{2}\right)=\mathrm{NV}\left(\sigma_{2}\right) \mathrm{NV}\left(\sigma_{3}\right)=M \mathrm{NV}\left(\sigma_{1}\right) \mathrm{NV}\left(\sigma_{3}\right)$, with $M \geq 2$. We thus get $\mathrm{NV}\left(\sigma_{3}\right)=\mathrm{NV}\left(\sigma_{1}\right)$ and $\mathrm{NV}\left(\sigma_{2}\right)=M \mathrm{NV}\left(\sigma_{1}\right)$ and we find then

$$
\operatorname{Aff}(\tau) \leftrightarrow x+M y+z=N(\tau)
$$

with $p(N(\tau), 0,0), q(0, N(\tau) / M, 0)$ and $r(0,0, N(\tau))$. In this case $e^{-\frac{2 \pi i}{N(\tau)}}$ is a zero of $F_{\tau}$ if and only if $\mathrm{NV}(\tau)+2>\mathrm{NV}\left(\sigma_{1}\right)+\mathrm{NV}\left(\sigma_{2}\right)+\mathrm{NV}\left(\sigma_{3}\right)$, or equivalently, if $\left(M \mathrm{NV}\left(\sigma_{1}\right)-2\right)\left(\mathrm{NV}\left(\sigma_{1}\right)-1\right)>0$. This is always the case, as $\mathrm{NV}\left(\sigma_{1}\right)=N(\tau) / M=1$ would imply that $\tau$ is a $B_{1}$-facet.

Case 4.3: If $N(\tau)=N\left(\sigma_{1} \cap \sigma_{2}\right), N(\tau) \neq N\left(\sigma_{1} \cap \sigma_{3}\right)$ and $N(\tau) \neq N\left(\sigma_{2} \cap \sigma_{3}\right)$, then by Proposition 5 one has $\mathrm{NV}(\tau)=\mathrm{NV}\left(\sigma_{1}\right) \mathrm{NV}\left(\sigma_{2}\right)=M_{1} \mathrm{NV}\left(\sigma_{1}\right) \mathrm{NV}\left(\sigma_{3}\right)=$ $M_{2} \mathrm{NV}\left(\sigma_{2}\right) \mathrm{NV}\left(\sigma_{3}\right)$, with $M_{1} \geq 2$ and $M_{2} \geq 2$. In this configuration we have

$$
\operatorname{Aff}(\tau) \leftrightarrow x+k y+l z=N(\tau),
$$

$p(N(\tau), 0,0), q(0, N(\tau) / k, 0)$ and $r(0,0, N(\tau) / l)$ with $\operatorname{gcd}(k, l)=1$. Then we find that $M_{1}=k, M_{2}=l$ and hence $\mathrm{NV}\left(\sigma_{2}\right)=k \mathrm{NV}\left(\sigma_{1}\right) / l$ and $\mathrm{NV}\left(\sigma_{3}\right)=\mathrm{NV}\left(\sigma_{1}\right) / l$. In this case, $e^{-2 \pi i / N(\tau)}$ would be a zero of $F_{\tau}$ if and only if $\mathrm{NV}(\tau)+1>\mathrm{NV}\left(\sigma_{1}\right)+\mathrm{NV}\left(\sigma_{2}\right)+\mathrm{NV}\left(\sigma_{3}\right)$, or equivalently,

$$
k \mathrm{NV}\left(\sigma_{1}\right)^{2}-(k+l+1) \mathrm{NV}\left(\sigma_{1}\right)+l>0 .
$$

This is true because $\mathrm{NV}\left(\sigma_{1}\right) \geq l$ while the largest real root of the polynomial on the left hand side is

$$
\frac{k+l+1+\sqrt{(k+l+1)^{2}-4 k l}}{2 k}<l
$$

the latter inequality holds because one easily rewrites it as $k l>k+l$, which holds since $k, l \geq 2$ and $k=l=2$ is excluded by coprimality.

Case 4.4: If $N(\tau) \neq N\left(\sigma_{1} \cap \sigma_{2}\right), N(\tau) \neq N\left(\sigma_{1} \cap \sigma_{3}\right)$ and $N(\tau) \neq N\left(\sigma_{2} \cap \sigma_{3}\right)$, then by Proposition 5 one has $\mathrm{NV}(\tau)=M_{1} \mathrm{NV}\left(\sigma_{1}\right) \mathrm{NV}\left(\sigma_{2}\right)=M_{2} \mathrm{NV}\left(\sigma_{1}\right) \mathrm{NV}\left(\sigma_{3}\right)=$ $M_{3} \mathrm{NV}\left(\sigma_{2}\right) \mathrm{NV}\left(\sigma_{3}\right)$, with $M_{1} \geq 2, M_{2} \geq 2$ and $M_{3} \geq 2$. In this configuration we have

$$
\operatorname{Aff}(\tau) \leftrightarrow k x+l y+m z=N(\tau),
$$

$p(N(\tau) / k, 0,0), q(0, N(\tau) / l, 0)$ and $r(0,0, N(\tau) / m)$ with $k, l, m$ pairwise coprime. Then we find that $M_{1}=k, M_{2}=l, M_{3}=m$ and hence $\mathrm{NV}\left(\sigma_{2}\right)=l \mathrm{NV}\left(\sigma_{1}\right) / m$ and $\mathrm{NV}\left(\sigma_{3}\right)=$ $k \mathrm{NV}\left(\sigma_{1}\right) / m$. In this case we want to establish that $\mathrm{NV}(\tau)>\mathrm{NV}\left(\sigma_{1}\right)+\mathrm{NV}\left(\sigma_{2}\right)+$ $\mathrm{NV}\left(\sigma_{3}\right)$, or equivalently that $k \operatorname{lm} \mathrm{NV}\left(\sigma_{1}\right)>k+l+m$. This follows from $\mathrm{NV}\left(\sigma_{1}\right) \geq 1$ and $k l m \geq 4 \max \{k, l, m\}>3 \max \{k, l, m\} \geq k+l+m$.

\subsection{On false poles contributed by $B_{1}$-facets and $X_{2}$-facets}

In [BV, Proposition 9.6] it is shown that if a candidate pole contributed only by $B_{1}$ facets is an actual pole of $Z_{f, 0}(\chi, s)$, then it is contributed by two $B_{1}$-facets w.r.t. different variables having a 1-dimensional intersection. We will here show that even in that situation the candidate pole is almost always a false pole of $Z_{f, 0}(\chi, s)$. We need this precision here, because for the holomorphy conjecture one has to verify if 
$1 / N_{j}$ gives rise to an eigenvalue of monodromy, rather than the quotient $\nu_{j} / N_{j}$ (that might be simplifiable). We also study when candidate poles of $Z_{f}(\chi, s)$ corresponding to $B_{1}$-facets are false poles. Finally we provide some facets of type $X_{2}$ that give rise to fake poles of $Z_{f, 0}(\chi, s)$ and $Z_{f}(\chi, s)$.

We again assume that the compact facets have been subdivided into simplices without introducing new vertices; this guarantees that every vertex is equipped with a nonzero coefficient. Then towards our study of contributions of configurations of $B_{1}$-facets, we make the following observations (which hold up to permutation of the coordinates).

FACT 1: A vertex $P(1, \cdot, \cdot)$ does not contribute. Indeed, as $\chi$ is not the trivial character (and so $\bar{\chi}$ neither is trivial), one immediately deduces from Lemma 8 that the contribution of $P$ is equal to 0 .

FACT 2: A vertex $P(a, 0,0)$ does not contribute if the order of $\bar{\chi}$ is not a divisor of $a$ (by Lemma 8 ).

FACT 3: A segment $\sigma:=P Q$ with $P(1,1, b)$ and $Q(0,0, a)$ does not contribute if the order of $\bar{\chi}$ is not a divisor of $a$. To compute the contribution of $\sigma$, we consider

$$
L_{\sigma}=q^{-3} \sum_{(x, y, z) \in\left(\mathbb{F}_{q}^{\times}\right)^{3}} \bar{\chi}\left(c_{0,0, a} z^{a}+c_{1,1, b} x y z^{b}\right) .
$$

By using Lemma 9, this expression simplifies to

$$
-q^{-3} \bar{\chi}\left(c_{0,0, a}\right) \sum_{(y, z) \in\left(\mathbb{F}_{q}^{\times}\right)^{2}} \bar{\chi}\left(z^{a}\right) .
$$

If the order of $\bar{\chi}$ is not a divisor of $a$, then it follows from Lemma 8 that the contribution of $\sigma$ is equal to 0 .

FACT 4: Let $\sigma:=P Q$ with $P(\cdot, \cdot, 0)$ and $Q(\cdot, \cdot, 0)$, and let $\tau:=P Q R$ with $R(\cdot, \cdot, 1)$ be the facet not contained in $\{z=0\}$ that contains $\sigma$, then $\sigma$ and $\tau$ cancel each other out. Indeed, by Lemma 9 with $f=f_{\sigma}$ it follows that $L_{\sigma}=(1-q) L_{\tau}$. As $\operatorname{mult}\left(\Delta_{\sigma}\right)=1$, we find that $L_{\sigma} S\left(\Delta_{\sigma}\right)+L_{\tau} S\left(\Delta_{\tau}\right)=0$.

FACT 5: Let $\sigma:=P Q$ with $P(\cdot, \cdot, 0)$ and $Q(\cdot, \cdot, 1)$, then again by Lemma 9 one finds $L_{P}=(1-q) L_{\sigma}$. Now let $\tau_{1}$ and $\tau_{2}$ be the facets containing $\sigma$ and let $\tau_{0}$ be the facet in $\{z=0\}$ containing the vertex $P$. With $\delta_{P}$ the cone (strictly positively) spanned by $\Delta_{\tau_{0}}, \Delta_{\tau_{1}}$ and $\Delta_{\tau_{2}}$ we then find that $L_{\sigma} S\left(\Delta_{\sigma}\right)+L_{P} S\left(\delta_{P}\right)=0$.

FACT 6: Let $\sigma:=P Q$ with $P(\cdot, \cdot, 0)$ and $Q(\cdot, \cdot, 1)$, and let $\tau_{1}$ be a non-compact $B_{1}$-facet containing $\sigma$. Let $\tau_{2}$ be the non-compact facet containing the vertex $Q$ and sharing a half line with $\tau_{1}$. Lemma 8 implies that $\tau_{1} \cap \tau_{2}$ does not contribute in the formula for $Z_{f}(\chi, s)$.

FACT 7: Let $\sigma:=P Q$ with $P(\cdot, \cdot, 0)$ and $Q(\cdot, \cdot, 1)$, and let $\tau_{1}$ be a non-compact $B_{1}$-facet containing $\sigma$. Let $\tau_{0}$ be the non-compact facet containing the vertex $P$ and sharing 
a half line $\sigma_{1}$ with $\tau_{1}$. As $L_{\sigma_{1}}=(1-q) L_{\tau_{1}}$ and mult $\left(\Delta_{\sigma_{1}}\right)=1$, it follows that the contributions of $\tau_{1}$ and $\sigma_{1}$ cancel each other out.

From these facts one can derive the contributions of all possible configurations of $B_{1}$ facets. We begin with the configuration studied (in the local case over $\mathbb{Q}_{p}$ ) in $[\mathrm{BV}$, Proposition 9.6] that we mentioned at the beginning of this section.

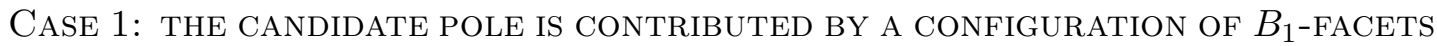
IN WHICH NO TWO FACETS THAT SHARE A 1-DimENSIONAL FACE ARE $B_{1}$ ONLY FOR DIFFERENT VARIABLES.

For the contributions to the local Igusa zeta function, one can derive from Facts 1, 4 and 5 that the candidate pole is a false pole. For the global Igusa zeta function, one in addition uses Facts 6 and 7.

Case 2: the Candidate pole is CONTRibuted by exactly two COMpaCt $B_{1}$ FACETS W.R.T. DIFFERENT VARIABLES, HAVING A LINE SEGMENT IN COMMON.

If the common line segment is compact, then the configuration is as in Figure 1 with

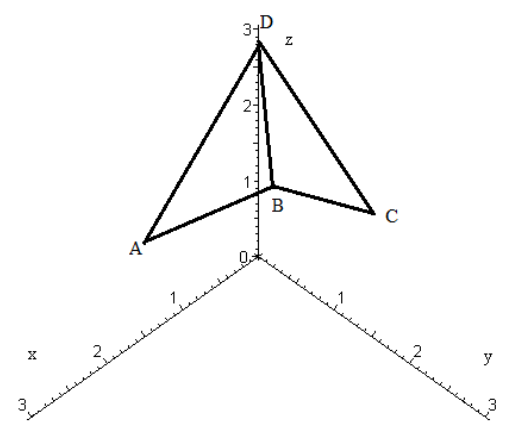

Figure 1

$A(., 0,),. B(1,1, b), C(0, .,$.$) and D(0,0, a)$. If the order of $\bar{\chi}$ is not a divisor of $a$, then it follows from Facts 1 to 5 that the candidate pole is a false pole of $Z_{f, 0}(\chi, s)$ and $Z_{f}(\chi, s)$.

Case 3: the Candidate pole is CONTRIbuted by two non-COMPaCt $B_{1}$-Facets W.R.T. DIFFERENT VARIABLES, HAVING A LINE SEGMENT IN COMMON.

If the common line segment is non-compact, then the configuration is as in Figure 2 , with $A(., 0,),. B(0, . .$.$) and C(1,1,$.$) . For the contributions to the local Igusa zeta$ function, one deduces from Facts 1, 4 and 5 that the candidate pole is a false pole. For the global Igusa zeta function, one also has to use Facts 6 and 7.

If the common line segment is compact, then its vertices are given by $A(0,0, a)$ and $B(1,1, b)$. If the order of $\bar{\chi}$ is not a divisor of $a$, then by Facts 1 to 7 it follows again that the candidate pole is not an actual pole of $Z_{f, 0}(\chi, s)$ and $Z_{f}(\chi, s)$. 


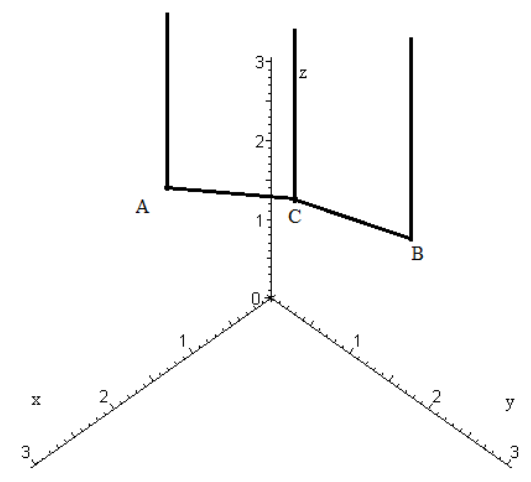

Figure 2

Case 4: the Candidate pole is CONTRibuted By one COMPaCt $B_{1}$-Facet and ONE NON-COMPACT $B_{1}$-FACETS W.R.T. DIFFERENT VARIABLES, HAVING A LINE SEGMENT IN COMMON.

Again using Fact 1 to Fact 7 , one finds that the candidate pole is a false pole of $Z_{f, 0}(\chi, s)$ and $Z_{f}(\chi, s)$ when the order of $\bar{\chi}$ is not a divisor of $a$,

Case 5: the Candidate pole is Contributed by at least two $B_{1}$-Facets W.R.T. DIFFERENT VARIABLES, HAVING A LINE SEGMENT IN COMMON.

As in Case 2 the contributions of $\tau_{1}:=A B D, \tau_{2}:=B C D$ and $\tau_{1} \cap \tau_{2}:=B D$ are all equal to 0 , one can deduce the fakeness of the candidate pole also when there are other $B_{1}$-facets having a 1-dimensional intersection with $\tau_{1}$ or $\tau_{2}$.

We now treat the candidate poles contributed by $X_{2}$-facets.

Lemma 13. Let $\tau$ be a facet with vertices $p(N(\tau) / 2,0,0), q\left(x_{1}, 0,2\right)$ and $r\left(x_{2}, 2,0\right)$ where $N(\tau) / 2-x_{1}$ and $N(\tau) / 2-x_{2}$ are odd. If the order of $\bar{\chi}$ does not divide $N(\tau) / 2$ and is different from 2 , then $\tau$ does not contribute to $Z_{f, 0}(\chi, s)$ and $Z_{f}(\chi, s)$.

Proof. It follows immediately from Fact 2 that the vertices $p, q$ and $r$ do not contribute. Using Lemma 7 one also verifies that the edge $q r$ does not contribute. We now show that the contributions of $\sigma_{1}:=p q, \sigma_{2}:=p r$ and the facet $\tau$ cancel each other. As $N\left(\sigma_{1}\right)=N\left(\sigma_{2}\right)=N(\tau)$, we have that mult $\left(\Delta_{\sigma_{1}}\right)=\operatorname{mult}\left(\Delta_{\sigma_{2}}\right)=1$, and thus

$$
S\left(\Delta_{\sigma_{i}}\right)=\frac{1}{(q-1)\left(q^{N(\tau) s+\nu(\tau)}-1\right)}, \quad 1 \leq i \leq 2 .
$$

One gets

$$
\begin{gathered}
L_{\sigma_{1}} S\left(\Delta_{\sigma_{1}}\right)+L_{\sigma_{2}} S\left(\Delta_{\sigma_{2}}\right)+L_{\tau} S\left(\Delta_{\tau}\right)=0 \\
\text { }) \\
(q-1) L_{\tau}=-L_{\sigma_{1}}-L_{\sigma_{2}} .
\end{gathered}
$$


The equality between these character sums is proven in Proposition 10.

If $x_{1}=x_{2}=0$ (in which case the $X_{2}$-facet is the only compact facet of $\Gamma_{0}$ ) we can prove something slightly stronger.

Lemma 14. Let $\tau$ be a facet with vertices $p(N(\tau) / 2,0,0), q(0,0,2)$ and $r(0,2,0)$ where $N(\tau) / 2$ is odd. If the order of $\bar{\chi}$ does not divide $N(\tau) / 2$, then $\tau$ does not contribute to $Z_{f, 0}(\chi, s)$ and $Z_{f}(\chi, s)$.

Proof. The previous proof remains valid, except for the conclusions that $q, r$ and $\sigma_{3}:=q r$ do not contribute, where we used that the order of $\bar{\chi}$ is not 2 . We show that the contributions cancel. Indeed, since $\operatorname{mult}\left(\Delta_{q}\right)=\operatorname{mult}\left(\Delta_{r}\right)=\operatorname{mult}\left(\Delta_{\sigma_{3}}\right)=N(\tau) / 2$ we have

$$
S\left(\Delta_{\sigma_{3}}\right)=\frac{N}{(q-1)\left(q^{N(\tau) s+\nu(\tau)}-1\right)}, \quad S\left(\Delta_{q}\right)=S\left(\Delta_{r}\right)=\frac{N}{(q-1)^{2}\left(q^{N(\tau) s+\nu(\tau)}-1\right)}
$$

for some common numerator $N$. One gets

$$
\begin{gathered}
L_{q} S\left(\Delta_{q}\right)+L_{r} S\left(\Delta_{r}\right)+L_{\sigma_{3}} S\left(\Delta_{\sigma_{3}}\right)=0 \\
\mathbb{\Downarrow} \\
(q-1) L_{\sigma_{3}}=-L_{q}-L_{r} .
\end{gathered}
$$

This again follows from Proposition 10 (with $\alpha=0$ ).

\subsection{The holomorphy conjecture for nondegenerate surface singulari- ties}

Theorem 15. Let $F$ be a number field and let $f(x, y, z) \in \mathcal{O}_{F}[x, y, z]$ be a polynomial which is nondegenerate over $\mathbb{C}$ w.r.t. the compact faces (resp. the faces) of its Newton polyhedron at the origin $\Gamma_{0}$. Let $K$ be a non-archimedean completion of $F$ with valuation ring $R$ (with maximal ideal $P$ ) and residue field $\mathbb{F}_{q}$, and suppose that $\bar{f}:=f \bmod P$ is nondegenerate over $\mathbb{F}_{q}$ w.r.t. the compact faces (resp. the faces) of $\Gamma_{0}$. Let $\chi$ be a nontrivial character of $R^{\times}$which is trivial on $1+P$. Let $\tau$ be a facet of $\Gamma_{0}$. If $-\nu(\tau) / N(\tau)$ is the real part of a pole of $Z_{f, 0}(\chi, s)$ (resp. $Z_{f}(\chi, s)$ ), then the order of $\chi$ divides the order of an eigenvalue of monodromy at some point of $f^{-1}\{0\}$.

Proof. As before we assume that all compact facets have been subdivided into simplices, without introducing new vertices. We first suppose that $\tau$ is such a simplex. If every 1-dimensional $\mathrm{V}$-face of $\Gamma_{0}$ is contained in a compact facet, then we know from Formula (3) that the zeta function of monodromy at the origin is a product of polynomials. If $\tau$ is not of type $B_{1}$ nor of type $X_{2}$, then Proposition 12 implies that the order of $\chi$ divides the order of an eigenvalue of monodromy of $f^{-1}\{0\}$ at the origin.

If $\tau$ is of type $B_{1}$, then we found in Subsection 5.2 that there is a point $p(0,0, a)$ in the configuration that is not the intersection of two 1-dimensional V-faces in a same compact facet, and secondly that the order of $\bar{\chi}$ divides this $a$. This means that the factor $1-t^{a}$ appears in $\zeta_{f, 0}(t)$ and so one finds that the order of $\chi$ divides the order of some eigenvalue of monodromy of $f^{-1}\{0\}$ at the origin.

If $\tau$ is of type $X_{2}$, say with vertices $p(N(\tau) / 2,0,0), q\left(x_{1}, 0,2\right)$ and $r\left(x_{2}, 2,0\right)$, then $F_{\tau}=1-t^{N(\tau) / 2}$ and hence $e^{-2 \pi i /(N(\tau) / 2)}$ is an eigenvalue of monodromy of $f^{-1}\{0\}$ at 
the origin. Thus if the order of $\bar{\chi}$ divides $N(\tau) / 2$ then we are done. If the order of $\bar{\chi}$ does not divide $N(\tau) / 2$, then by Lemma 13 the order of $\bar{\chi}$ should be equal to 2 . In this situation $N(\tau) / 2$ is odd and $x_{1}$ and $x_{2}$ are even, while by Lemma 14 we can assume that $0 \neq x_{1} \geq x_{2}$. Let $\tau^{\prime}$ be the other facet which contains the segment $q r$. Notice that $N\left(\tau^{\prime}\right)$ is even and that $\tau^{\prime}$ is not of type $B_{1}$. We first suppose that $\tau^{\prime}$ is compact. If $\tau^{\prime}$ is not of type $X_{2}$, then it follows from Proposition 12 that $e^{-2 \pi i / N\left(\tau^{\prime}\right)}$ is a zero of $F_{\tau^{\prime}}$ and so the order of $\chi$ divides the order of some eigenvalue of monodromy of $f^{-1}\{0\}$ at the origin. If $\tau^{\prime}$ is of type $X_{2}$, then the configuration is as in Figure 3.

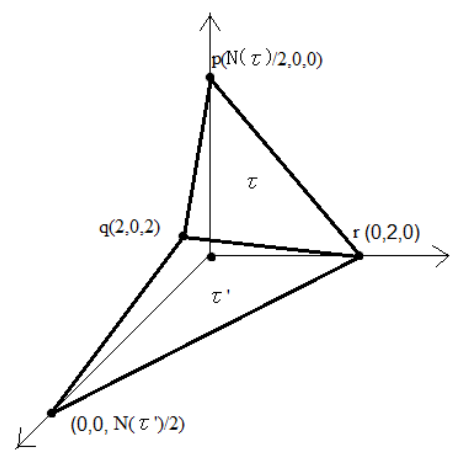

Figure 3

In this situation, we get

$$
\zeta_{f, 0}(t)=\left(1-t^{N(\tau) / 2}\right)\left(1-t^{N\left(\tau^{\prime}\right) / 2}\right)\left(1-t^{2}\right),
$$

and so again the order of $\chi$ divides the order of an eigenvalue of monodromy of $f^{-1}\{0\}$ at the origin.

Suppose now that $\tau^{\prime}$ is not compact, then necessarily $x_{1}>x_{2}$ and

$$
\operatorname{Aff}\left(\tau^{\prime}\right) \leftrightarrow x+\frac{x_{1}-x_{2}}{2} y=x_{1} .
$$

At a generic point $(0,0, c)$ of the hypersurface, the polynomial $g(x, y, z):=f(x, y, z-c)$ is still nondegenerate w.r.t. the compact faces of its Newton polyhedron at the origin (see Lemma 21) and its Newton polyhedron is the projection onto $\{z=0\}$ of the Newton polyhedron of $f$ times $\mathbb{R}_{+}$. From Varchenko's formula one sees that this projected polyhedron fully determines $\zeta_{g, 0}(t)$. Using [LVP2, Proposition 5] it follows that $\zeta_{g, 0}(t)$ contains the factor $1 /\left(1-t^{x_{1}}\right)$. We thus have that the order of $\chi$ divides the order of an eigenvalue of monodromy at a point of the hypersurface in the neighbourhood of the origin.

Suppose now that there is a 1-dimensional V-face $\sigma$, say in the coordinate plane $z=0$, which is not contained in a compact facet. If $e^{-2 \pi i / N(\tau)}$ is a zero of $F_{\sigma}$ (we use the notation $F_{\sigma}$ as if $\sigma$ was a facet of a two-dimensional Newton polyhedron in the plane $z=0)$, then we choose $c \in \mathbb{C}$ close to zero such that $g(x, y, z):=f(x, y, z-c)$ is still nondegenerate w.r.t. its Newton polyhedron at the origin (see Lemma 2). Then we have $\zeta_{g, 0}(t)=\prod_{\sigma \text { compact facet }} F_{\sigma}$, with $F_{\sigma}=1 /$ polynomial (except the case where $\sigma$ 
contains two vertices on coordinate axes, but in this case the same conclusion holds) and so we find that $e^{-2 \pi i / N(\tau)}$ is an eigenvalue of monodromy of $f$ at $(0,0, c)$.

Finally let $\tau$ be non-compact. Again by the nondegeneracy argument (Lemma 21) we can reduce the dimension and conclude that $e^{-2 \pi i / N(\tau)}$ is an eigenvalue of monodromy of $f$ at a point in the neighbourhood the origin.

\section{References}

[AVG] V. Arnold, A. Varchenko and S. Goussein-Zadé, Singularités des applications différentiables II, Editions Mir, Moscou (1986).

[BV] B. Bories and W. Veys, Igusa's p-adic local zeta function and the monodromy conjecture for non-degenerated surface singularities, Memoirs of the American Mathematical Society, (to appear), 119p.

[D1] J. Denef, Local zeta functions and Euler characteristics, Duke Math. J. 63 (1991), 713-721.

[D2] J. Denef, Report on Igusa's local zeta function, Séminaire Bourbaki 43 (19901991), exp. 741; Astérisque 201-202-203 (1991), 359-386.

[D3] J. Denef, Degree of local zeta functions and monodromy, Comp. Math. 89 (1993), 207-216.

[DL] J. Denef and F. Loeser, Caractéristique d'Euler-Poincaré, fonctions zêta locales et modifications analytiques, J. Amer. Math. Soc. 5, 4 (1992), 705-720.

[DV] J. Denef and W. Veys, On the holomorphy conjecture for Igusa's local zeta function, Proc. Amer. Math. Soc. 123 (1995), 2981-2988.

[H] K. Hoornaert, Newton polyhedra and the poles of Igusa's local zeta function, Bull. Belg. Math. Soc. - Simon Stevin, 9 no.4 (2002), 589-606.

[I] J. Igusa, Complex powers and asymptotic expansions I, J. Reine Angew. Math. 268/269 (1974) 110-130; II, ibid. 278/279 (1975), 307-321.

[LVP1] A. Lemahieu and L. Van Proeyen, The holomorphy conjecture for ideals in dimension two, Proc. Amer. Math. Soc. 139 (2011), 3845-3852.

[LVP2] A. Lemahieu and L. Van Proeyen, Monodromy conjecture for nondegenerate surface singularities, Trans. Amer. Math. Soc. 363 (2011), no.9, 4801-4829.

[LV] A. Lemahieu and W. Veys, Zeta functions and monodromy for surfaces that are general for a toric idealistic cluster, Int. Math. Res. Notices, 1 (2009), 11-62.

[RV] B. Rodrigues and W. Veys, Holomorphy of Igusa's and topological zeta functions for homogeneous polynomials, Pac. J. of Math. 201 (2001), 429-440.

[ST] K. Smith and H. Thompson, Irrelevant exceptional divisors for curves on a smooth surface, Algebra, geometry and their interactions, Contemp. Math., vol. 448, Amer. Math. Soc., Providence, RI, 2007, pp. 245-254. 
[Va] A. Varchenko, Zeta-function of monodromy and Newton's diagram, Inventiones Math. 37 (1976), 253-262.

[Ve] W. Veys, Holomorphy of local zeta functions for curves, Math. Annalen 295 (1993), 635-641.

[W] A. Weil, Sur les courbes algébriques et les variétés qui s'en déduisent, Hermann, Paris, 1948.

Vakgroep Wiskunde, Universiteit Gent

Krijgslaan 281, 9000 Gent, Belgium

Departement Elektrotechniek, KU Leuven

Kasteelpark Arenberg 10/2452, 3001 Leuven, Belgium

E-mail address: wouter.castryck@gmail.com

Faculty of Mathematics and Informatics, Ovidius University

BD. Mamaia 124, 900527 Constanta, Romania

E-mail address: denis@univ-ovidius.ro

Laboratoire Paul Painlevé, Université Lille 1

Cité Scientifique, 59655 Villeneuve d'Ascq Cedex, France

E-mail address: lemahieu.ann@gmail.com 